\title{
Mapping evidence on ovarian, endometrial, vaginal, and vulva cancer research in Africa: a scoping review protocol
}

\author{
Sebastian Yidana Ninimiya ${ }^{1,2}$, Monica Ansu-Mensah ${ }^{3}$, Vitalis Bawontuo ${ }^{1,4}$ and Desmond Kuupiel ${ }^{4,5^{*}}$ (D)
}

\begin{abstract}
Background: Globally, cancer is generally recognized as a developmental threat yet most countries in Africa lack capacity to diagnose cancer especially gynecological cancers resulting in late detection and poor outcomes. However, most studies on gynecological cancers in Africa tend to focus on cervical cancer compared to the other gynecological cancers. Therefore, this scoping review will aim to describe the existing literature on the epidemiological burden of ovarian, endometrial, vaginal, and vulva cancers, their risk factors, and potential screening methods/techniques in Africa to identify priority research gaps for further research to inform health policy decisions.
\end{abstract}

Methods: The framework promulgated by Arksey and O'Malley and improved by Levac et al. will be used as a guide for this scoping review. A comprehensive search for relevant published studies in PubMed, CINAHL, SCOPUS, Google Scholar, and ScienceDirect with no date limitation to the last search date. The database search strategy will include keywords, Boolean operators, and medical subject heading terms. We will additionally consult the WHO/ IARC website, IHME/Global Burden of Disease Study. A snowball approach will also be used to search the reference list of all included studies to obtain relevant papers for possible inclusion in this review. We will include articles that involve African countries, focused on ovarian, endometrial, vaginal, and vulva cancers, their risk factors, and potential screening methods/techniques in any language. We will exclude studies on cervical cancer and other cancers as well as review articles. The abstracts and full-text selection will be conducted by two independent reviewers using this review's eligibility criteria as a guide. All the review selection tools, and the data extraction form will be pilot tested for accuracy and consistency. The data will be organized into thematic areas, summarized and the results communicated narratively.

Discussion: It is anticipated that this review will reveal important literature gaps to guide future research to inform health policy decisions about ovarian, endometrial, and rare gynecological neoplasms in Africa. This review's findings will be disseminated via peer review journals, conferences, and other social media such Twitter and Linkedln.

Keywords: Gynecological cancer, Ovarian, Endometrial, Rare gynecological neoplasms, Screening techniques, Epidemiological burden, Risk factors, Africa

\footnotetext{
* Correspondence: desmondkuupiel98@hotmail.com

${ }^{4}$ Centre for Evidence-based Health Care, Division of Epidemiology and Biostatistics, Department of Global Health, Faculty of Medicine and Health

Sciences, Stellenbosch University, Cape Town 7530, South Africa

${ }^{5}$ Research for Sustainable Development ( $\left.r 4 \mathrm{ds}\right)$ consult, Sunyani, Ghana

Full list of author information is available at the end of the article
}

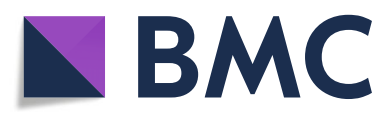

(c) The Author(s). 2021 Open Access This article is licensed under a Creative Commons Attribution 4.0 International License, which permits use, sharing, adaptation, distribution and reproduction in any medium or format, as long as you give appropriate credit to the original author(s) and the source, provide a link to the Creative Commons licence, and indicate if changes were made. The images or other third party material in this article are included in the article's Creative Commons licence, unless indicated otherwise in a credit line to the material. If material is not included in the article's Creative Commons licence and your intended use is not permitted by statutory regulation or exceeds the permitted use, you will need to obtain permission directly from the copyright holder. To view a copy of this licence, visit http://creativecommons.org/licenses/by/4.0/. The Creative Commons Public Domain Dedication waiver (http://creativecommons.org/publicdomain/zero/1.0/) applies to the data made available in this article, unless otherwise stated in a credit line to the data. 


\section{Background}

Cancer is generally recognized as a threat to global development. In 2017, the global incidence and deaths due to cancer were estimated to be approximately 24.5 million cases and 9.6 million deaths respectively [1]. Cancer is part of the emerging non-communicable diseases in Africa with an ever-increasing burden on individuals, communities, and healthcare systems. A higher number of people die from cancers compared to acquired immunodeficiency syndrome, tuberculosis, and malaria combined [2]. Cancer (including gynecological cancers) hitherto was thought to be a disease of the affluent particularly those in high-income countries, but there are indications that the burden of cancer is rising in lowand-middle-income countries (LMICs) despite facing diagnostic challenges [2]. In 2018, the global cancer incidence, mortality, and prevalence, number (GLOBOCAN) report showed a steady rise in the occurrence of gynecological cancers in LMICs [3].

Gynecological cancers include cervical cancer, endometrial cancer, ovarian and fallopian tube cancers, cancer of the vagina, and cancer of the vulva. Primary vaginal cancer is relatively rare but is associated with HIV infection which is highly prevalent in Africa [4]. Most vaginal cancers are secondary, but chronic human papillomavirus (HPV) infections which are rife in Africa results in primary vaginal cancer [4, 5]. Vulva cancer also relatively rare has a causal relationship with chronic oncogenic HPV infections and preventive strategies aimed at cervical cancer has benefits for vulva cancer [6, 7]. Endometrial cancer is the third commonest gynecological cancer in LMICs [2]. The risk factors of these cancers may include a sedentary lifestyle, obesity, unopposed estrogen, infertility, and decreased fertility rates $[8,9]$. With a growing westernization in the lifestyle of Africans, these risk factors are increasingly becoming important in the continent. Ovarian cancer with about $18.8 \%$ representation of all gynecological cancers in LMICs [3] is characterized by a high case fatality rate because it is largely asymptomatic until the late stages of the disease [10]. Fallopian tube cancers have similar characteristics with ovarian cancers and are classified by the International Federation of Gynaecology and Obstetrics together with ovarian cancers [10].

A prior review indicated an increasing burden of gynecological cancers in LMICs due to a rise in cervical cancer incidence [11]. In 2013, Iyoke and Ugwu conducted a review and reported that $60 \%$ of gynecological cancers in these countries which are mostly found in Africa are due to cervical cancer [11]. The highest incidence and mortality of cervical cancers occur in African countries [4] mostly due to the absence of effective national preventive strategies in these countries [11]. Together with vaginal cancer and cancer of the vulva, cervical cancer is highly preventable with vaccination against the highly oncogenic HPV species [4, 6, 7, 12]. However, poor diagnostic abilities in most African countries coupled with general poor health-seeking behavior is likely to hamper early detection and desired treatment outcomes of cancer including most gynecological cancers. Although several gynecological cancers exist, much attention has been given to cervical cancer compared to the others particularly in Africa due to known reasons including diagnostic challenges. Nonetheless, research on the other gynecological neoplasms such as ovarian, endometrial, and other rare gynecological neoplasms is essential. Several previous reviews have been published in the past relating to gynecological cancer screening [11, 13-15] but to date, the range of research and knowledge gaps on ovarian, endometrial, vaginal, and vulva cancers in Africa is not known. Therefore, we propose to conduct a scoping review to describe evidence about gynecological cancers in Africa focusing on the epidemiological burden of ovarian, endometrial, vaginal, and vulva cancers, their risk factors, and potential screening methods/techniques. We anticipate that this review will reveal important literature gaps to inform future research to inform health policy decisions about ovarian, endometrial, and rare gynecological neoplasms in Africa.

\section{Methods}

\section{Overview of the study}

Scoping reviews aim to map rapidly the key concepts underpinning a research area and the main sources and types of evidence available. Scoping reviews also involve the synthesis and analysis of a wide range of research and non-research material to provide greater conceptual clarity about a specific topic or field of evidence [16-18]. Scoping reviews aim to provide a map of what evidence has been produced from disparate or heterogeneous sources as opposed to seeking only the best evidence to answer a particular question related to policy or practice [18]. This review will be based on the 2005 Arksey and O'Malley framework as reviewed and enhanced by Levac and colleagues in 2010 [19-21]. This protocol stipulates 5 mandatory and 1 optional steps as follows: identification of research question; identification of relevant studies; study selection; charting of data, collating, summarizing and reporting the results; and the optional step of consultation [20-22]. This protocol is reported according to the Preferred Reporting Items for Systematic Reviews and Meta-Analysis Protocol (PRISMA-P) statement [23]. However, the resulting paper will be reported per the PRISMA extension for Scoping Reviews checklist [21].

\section{Identifying the research question}

Our overall scoping review question will be: What evidence exists on ovarian, endometrial, vaginal, and 
vulva cancer research in Africa? The population, concept, and context (PCC) mnemonic (Table 1) was used to determine the eligibility of the review question. The secondary research questions include:

- What evidence exists on the epidemiological burden (morbidity, incidence, prevalence, and mortality) of ovarian, endometrial, vaginal, and vulva cancers in Africa?

- What evidence exists on the risk factors of ovarian, endometrial, vaginal, and vulva cancers in Africa?

- What evidence exists on potential screening techniques/methods/recommendations for ovarian, endometrial, vaginal, and vulva cancers in Africa?

\section{Information sources and search strategy}

A comprehensive search for relevant published articles on gynecological cancers in Africa will be conducted in the following databases: PubMed/MEDLINE, CINAHL, Web of science, SCOPUS, Google Scholar, and ScienceDirect without date limitation to the last search date. The search will include the use of a combination of relevant keywords and index terms with appropriate Boolean operators (AND/OR). Medical Subject Headings $(\mathrm{MeSH})$ terms or subject heading in all fields will be included and syntax modified appropriately for each database if necessary, to enable identification of all relevant studies. Study design and publication language limitations will be removed during the search. An electronic pilot search strategy for this review is illustrated in Table 2. Nonetheless, the authors will consult an experienced librarian to improve the search strategy if needed. We will also consult the websites of the WHO/International Agency for Research on Cancer, Institute for Health Metrics and Evaluation/Global Burden of Disease Study. A snowball approach will further be used to search the reference list of all included articles to obtain relevant articles for possible inclusion in this review. A detailed searched record will be documented as follows: date of search, search engine used, keywords used in the search, the number of retrieved publications, and the number of eligible studies found. The principal investigator (SYN) who is an expert obstetrician and gynecologist will conduct the search in the electronic databases. Mendeley Desktop reference manager will be used to compile all the articles deemed relevant for this review.

\section{Study selection and eligibility criteria Study selection}

The principal investigator (SYN) will conduct an extensive title screening guided by this review's eligibility criteria and all eligible studies imported to a folder created in Mendeley reference manager for this scoping review study. The library created will be cleaned by identifying and removing duplicate articles before the abstract screening phase. Subsequently, the cleaned library will be shared among the review team. SYN and MAM will independently screen the abstract and full text using the eligibility criteria for the review. Disagreements between SYN and MAM responses shall be resolved by dialog and consensus-building. DK will serve as an arbiter at the full-text screening stage if any disagreement arises between SYN and MAM. In the case where a full-text article cannot be retrieved or is not accessible from the online databases, assistance will be sought from the Catholic University College library or the Stellenbosch University to obtain it for screening. We will also consider writing to the authors to request any full-text article not accessible freely online if needed. Finally, this review will adopt the PRISMA flow chart [22, 23] (Fig. 1) to account for the articles at each stage of the screening.

\section{Eligibility criteria of this review}

Inclusion criteria This will include the following:

- Studies that involved African countries

- Studies that include human participants (women)

- Studies that include ovarian, endometrial, vaginal, and vulva cancers

- Studies reporting on the epidemiological burden (morbidity, incidence, prevalence, and mortality) of ovarian, endometrial, vaginal, and vulva cancers

- Studies that reported findings on the risk factors of ovarian, endometrial, vaginal, and vulva cancers

- Primary study designs (quantitative, qualitative, and mixed methods studies)

Exclusion criteria This will include the following:

- Studies that focused on other gynecological cancers such as cervical cancer

- Studies that focused on other types of cancer such as breast cancer

Table 1 PCC defining the eligibility of the main scoping review question

\begin{tabular}{ll}
\hline $\begin{array}{l}\text { P- } \\
\text { Population }\end{array}$ & Women \\
\hline C-Concept & Gynecological neoplasms: This will include ovarian, endometrial, vaginal, and vulva cancers \\
C-Context & $\begin{array}{l}\text { The epidemiological burden (incidence, morbidity, prevalence, and mortality); risks factors; and potential screening methods/ } \\
\text { techniques in African countries }\end{array}$ \\
\hline
\end{tabular}


Table 2 Pilot search for the review conducted on PubMed

\begin{tabular}{|c|c|c|c|}
\hline Date & Database & Keywords & $\begin{array}{l}\text { Search } \\
\text { results }\end{array}$ \\
\hline $\begin{array}{l}18 / 09 / \\
2020\end{array}$ & PubMed & 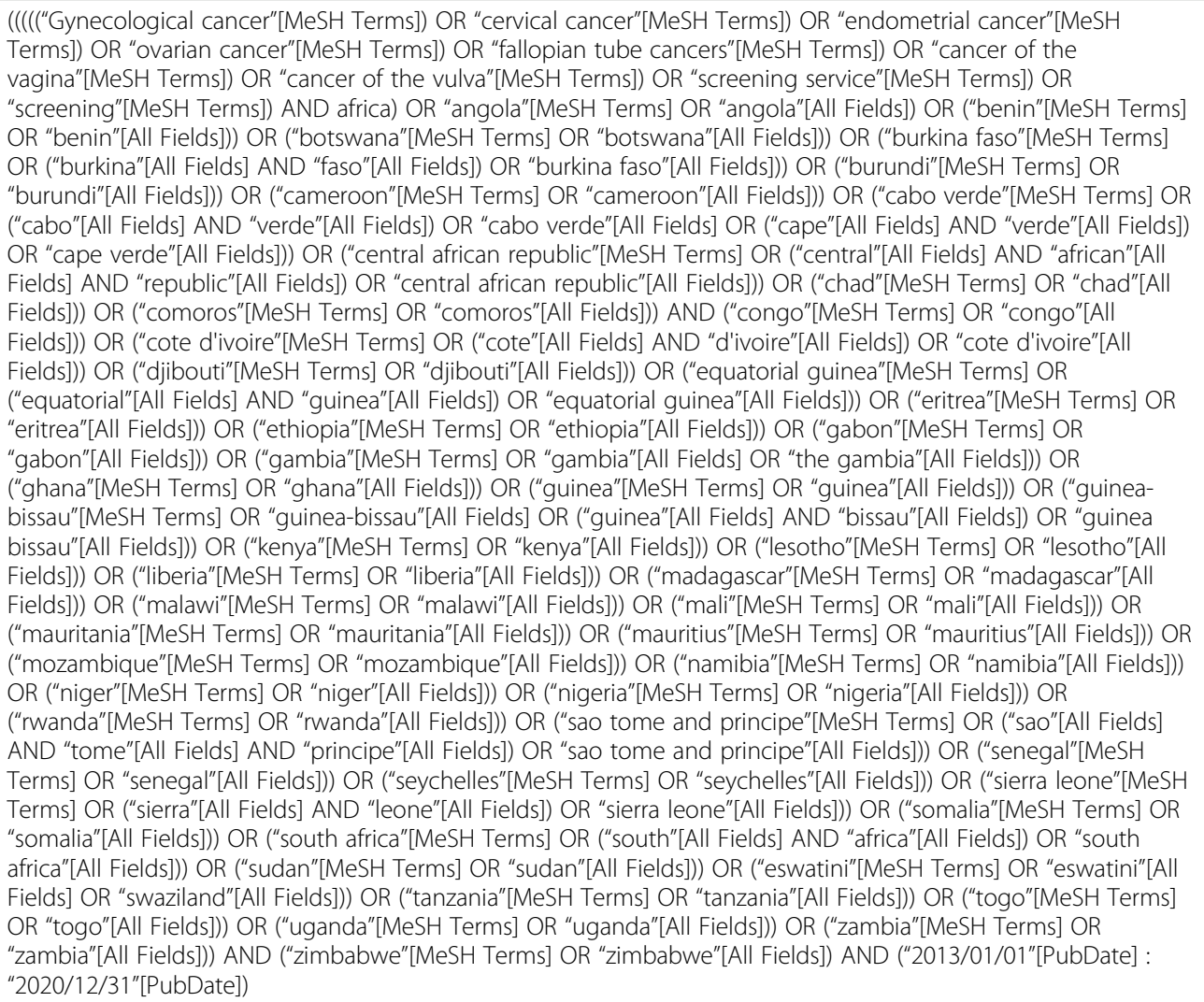 & 15538 \\
\hline
\end{tabular}

- Studies focus on the natural science of ovarian, endometrial, vaginal, and vulva cancers

- Studies focusing on the diagnostic and/ management/treatment of ovarian, endometrial, vaginal, and vulva cancers

- Studies focusing on the economic burden of ovarian, endometrial, vaginal, and vulva cancers

- Gray literature, conference papers, opinions, editorials, theses, dissertations, and unpublished studies

- Articles without full text

\section{Charting the data}

We will extract all the germane findings from the included articles aimed at answering the main scoping review question. Two reviewers (SYN and MAM) will independently pilot the data extraction form using 10 percent of the included studies to ensure consistency and uniformity. Appropriate adjustments will consequently be made for final use. The data extraction form will be updated regularly until all relevant and applicable information has been extracted. The data extraction form will include the following:
- Author and publication year

- Study title

- Aim/objective of the study

- Geographical location of the study (country)

- Type of study design

- Study setting (such as facility-based, communitybased, others)

- Number of study participants

- Mean age/range of study population (women)

- Targeted gynecological cancer

- Burden (incidence, prevalence, morbidity, mortality)

- Reported risk factors per the type of gynecological cancer investigated

- Potential screening techniques/methods/ recommendations

- Relevant recommendations/conclusions

\section{Collating, summarizing, and reporting the results}

Thematic content analysis will be employed to describe the themes that are related to this review's objective. The thematic analysis would ensure the identification of all the themes essential to address the research question. The sub-themes will be collated and structured around the following main themes: the epidemiological burden 


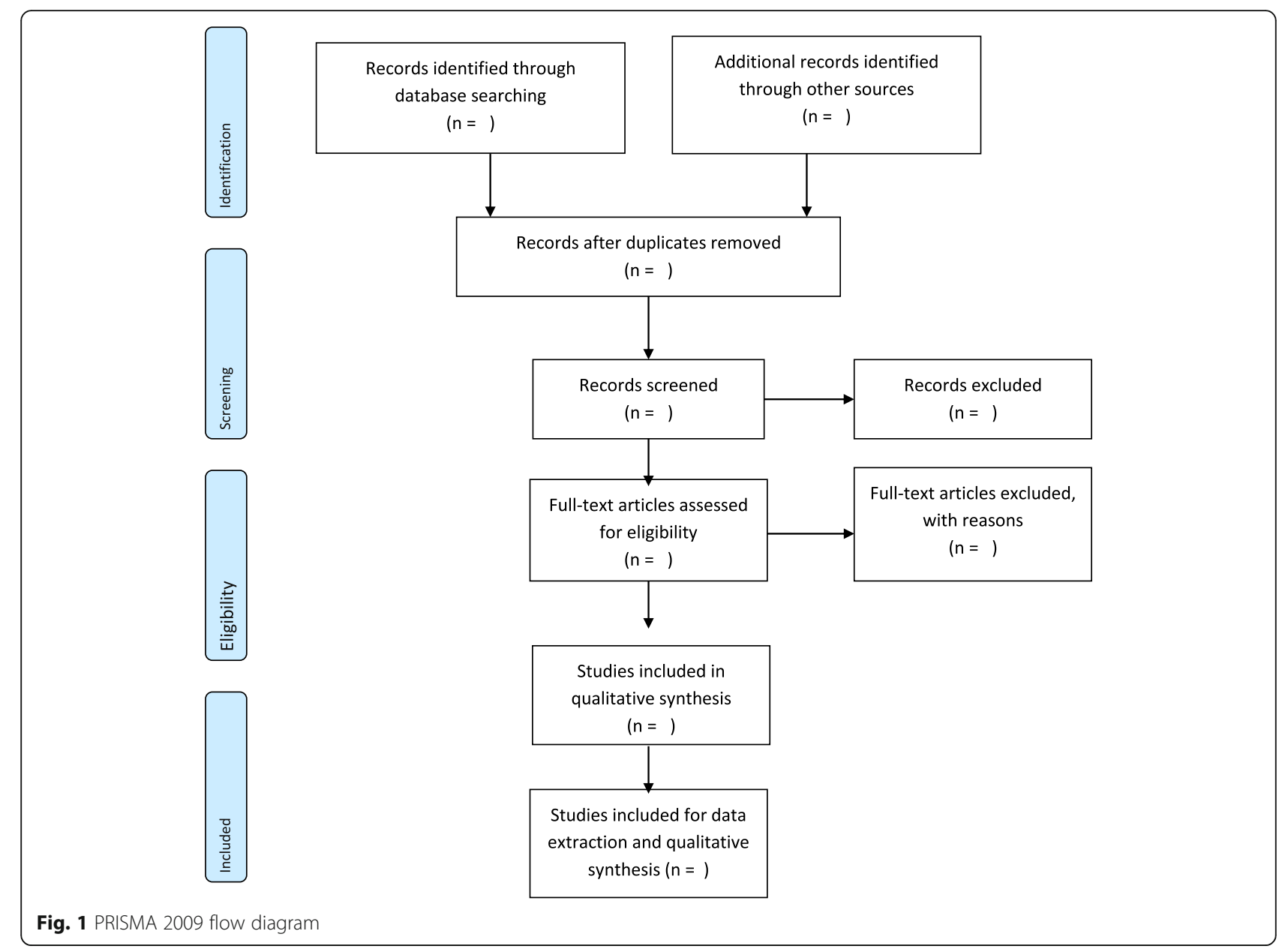

(incidence, morbidity, prevalence, and mortality) of ovarian, endometrial, vaginal, and vulva cancers in Africa; and the reported risk factors of ovarian, endometrial, vaginal, and vulva cancers in Africa; and potential screening methods/techniques for ovarian, endometrial, vaginal, and vulva cancers in Africa. This review will also analyze other relevant emerging themes. Subsequently, we will summarize the findings and a narrative account for each theme or outcome reported. A map will be used to show the geographical locations of the included studies. Graphs and tables will also be used to present the study characteristics such as the study designs, and others where possible. If feasible, a follow-up metaanalysis using quantitative data from this review will be considered due to the qualitative nature of scoping review studies.

\section{Discussion}

This scoping review study aims to describe the existing literature on the epidemiological burden of ovarian, endometrial, vaginal, and vulva cancers, their risk factors, and potential screening methods/techniques in
Africa. Cancer is a global health problem and remains a threat to development but many countries in low-andmiddle-income countries (LMICs) lack capacity to diagnose most cancers including gynecological cancers which often result in late detection and poor outcomes. Research relating to gynecological cancers in many LMICs tends to focus more on cervical cancer although several other gynecological cancers exist. Knowledge of the range of evidence or research activity on the other gynecological cancers aside from cervical cancer is essential to help identify priority research gaps for future research to facilitate health policy decisions, hence, the need for this review. Due to lack of external funding, this review will be limited to African countries only though the inclusion of other LMICs would have been great. Similarly, we will not cover research on diagnostic and/ management/treatment of ovarian, endometrial, vaginal, and vulva cancers as well as other rare gynecological cancers. The evidence obtained from this review will be disseminated via publication on several platforms including peer-reviewed journals, conferences, and interactions with potential knowledge users. 


\section{Abbreviations}

LMICs: Low-and-middle-income countries; HPV: Human papilloma virus: HIV: Human immunodeficiency virus; MMAT: Mixed methods appraisal tool

\section{Acknowledgements}

We are thankful to the Faculty of Health and Allied Health Sciences, Catholic University College, Fiapre, Sunyani, Ghana, for the diverse support leading to the conceptualization of this study.

\section{Authors' contributions}

SYN conceptualized the review, design the review methodology, and wrote the protocol under the supervision of DK. MAM contributed to the writing. DK and BV critically reviewed the draft and made revisions. SYN wrote the final draft and MAM, BV, and DK approved the final draft.

\section{Funding}

No funding has been obtained for the scoping review.

\section{Availability of data and materials}

We have duly cited all studies and data is presented in the form of references.

\section{Declarations}

Ethics approval and consent to participate

Not applicable.

\section{Consent for publication}

Not applicable.

\section{Competing interests}

We declare there are no competing interests.

\section{Author details}

${ }^{1}$ Faculty of Health and Allied Sciences, Catholic University College of Ghana, Fiapre, Sunyani, Ghana. ${ }^{2}$ St. Theresa's Hospital, Nandom, Upper West Region, Ghana. ${ }^{3}$ Sunyani Technical University, Sunyani, Bono Region, Ghana. ${ }^{4}$ Centre for Evidence-based Health Care, Division of Epidemiology and Biostatistics, Department of Global Health, Faculty of Medicine and Health Sciences, Stellenbosch University, Cape Town 7530, South Africa. ${ }^{5}$ Research for Sustainable Development (r4ds) consult, Sunyani, Ghana.

Received: 28 September 2020 Accepted: 29 March 2021 Published online: 14 April 2021

\section{References}

1. Fitzmaurice C, Abate D, Abbasi N, Abbastabar H, Abd-Allah F, AbdelRahman O, et al. Global, regional, and national cancer incidence, mortality, years of life lost, years lived with disability, and disability-Adjusted life-years for 29 cancer groups, 1990 to 2017: a systematic analysis for the global burden of disease study. JAMA Oncol. 2019;5(12):1749-68. https://doi.org/1 0.1001/jamaoncol.2019.2996

2. Beaulieu N, Bloom DE, Bloom/ LR, Stein RM. Breakaway: The global burden of cancer - 2009

3. Bray F, Ferlay J, Soerjomataram I. Global Cancer Statistics 2018: GLOBOCAN estimates of incidence and mortality worldwide for 36 cancers in 185 countries; 2018. p. 394-424

4. Adams TS, Cuello MA. Cancer of the vagina. Int J Gynecol Obstet. 2018;143: 14-21. https://doi.org/10.1002/ijgo.12610.

5. Krings A, Dunyo P, Pesic A, Tetteh S, Hansen B, Gedzah I, et al. Characterization of human papillomavirus prevalence and risk factors to guide cervical cancer screening in the North Tongu District, Ghana. Plos One. 2019;14(6):1-19.

6. Nygård $M$, Hansen BT, Dillner J, Munk C, Oddsson $K$, Tryggvadottir L, Hortlund M, Liaw KL, Dasbach EJ, Kjær SK. Targeting human papillomavirus to reduce the burden of cervical, vulvar and vaginal cancer and pre-invasive neoplasia: establishing the baseline for surveillance. PLoS One. 2014;9(2): e88323.

7. Rogers L, Cuello MA. Cancer of the vulva. Int J Gynecol Obstet. 2018;143:413. https://doi.org/10.1002/ijgo.12609.
8. Botha MH. Endometrial carcinoma: a South African perspective. South African J Gynaecol Oncol. 2009;1(1):16-20. https://doi.org/10.1080/2074283 5.2009 .11441129$.

9. Amant F, Mirza MR, Koskas M, Creutzberg CL. Cancer of the corpus uteri. Int J Gynecol Obstet. 2018;143:37-50. https://doi.org/10.1002/ijgo.12612.

10. Berek JS, Kehoe ST, Kumar L, Friedlander M. Cancer of the ovary, fallopian tube, and peritoneum. Int J Gynecol Obstet. 2018;143:59-78. https://doi. org/10.1002/ijgo. 12614 .

11. lyoke CA, Ugwu GO. Burden of gynecological cancers in developing countries. World J Obstet Gynaecol. 2013;2(1):5.

12. Bhatla N, Aoki D, Sharma DN, Sankaranarayanan R. Cancer of the cervix uteri. Int J Gynecol Obstet. 2018;143:22-36. https://doi.org/10.1002/ ijgo.12611.

13. Runowicz CD, Fields AL. Screening for gynecologic malignancies: a continuing responsibility. Surg Oncol Clin N Am. 1999:8(4):703-23. https:// doi.org/10.1016/S1055-3207(18)30184-4.

14. Sharma A, Menon U. Screening for gynaecological cancers. Eur I Surg Oncol. 2006;32(8):818-24. https://doi.org/10.1016/j.ejso.2006.03.034.

15. Hall KL, Dewar MA, Perchalski J. Screening for gynecologic cancer. Vulvar, vaginal, endometrial, and ovarian neoplasms. Prim Care. 1992;19(3):607-20.

16. Pham MT, Greig JD, Sargeant JM, Mcewen SA. A scoping review of scoping reviews: advancing the approach and enhancing the consistency. 2015;(July 2014)

17. Colquhoun $\mathrm{H}$, Science $\mathrm{O}$, Therapy O. Current best practices for the conduct of scoping; 2016.

18. Cacchione PZ. The evolving methodology of scoping reviews; 2016.

19. Kalisa R, Rulisa $S$, van den Akker T, van Roosmalen J. Maternal near miss and quality of care in a rural Rwandan hospital. BMC Pregnancy Childbirth. 2016; 16(1):1-8.

20. Arksey H, Malley LO. Scoping studies: towards a methodological framework; 2005. p. 19-32.

21. Levac D, Colquhoun H, Brien KKO. Scoping Studies: advancing the methodology scoping studies: advancing the methodology. 2010;(March 2015).

22. Appendix 1: methodological framework ( Arksey \& O'Malley, 2005 ). 2013; 2013.

23. Information A. PRISMA-P (Preferred Reporting Items for Systematic Review and Meta-Analysis Protocols ) 2015 checklist: recommended items to address in a systematic review protocol *. 2015;

\section{Publisher's Note}

Springer Nature remains neutral with regard to jurisdictional claims in published maps and institutional affiliations.

Ready to submit your research? Choose BMC and benefit from:

- fast, convenient online submission

- thorough peer review by experienced researchers in your field

- rapid publication on acceptance

- support for research data, including large and complex data types

- gold Open Access which fosters wider collaboration and increased citations

- maximum visibility for your research: over $100 \mathrm{M}$ website views per year

At $\mathrm{BMC}$, research is always in progress.

Learn more biomedcentral.com/submission 